\title{
O mercado de planos e seguros de saúde no Brasil: uma abordagem exploratória sobre a estratificação das demandas segundo a PNAD 2003
}

\author{
The market of health plans and insurances in Brazil: \\ an exploratory approach to the stratification of demands \\ based on the 2003 PNAD
}

\author{
Ligia Bahia \\ Ronir Raggio Luiz \\ Cláudio Salm ${ }^{3}$ \\ Antonio José Leal Costa ${ }^{2}$ \\ Pauline Lorena Kale ${ }^{2}$ \\ Maria de Lourdes T. Cavalcanti ${ }^{1}$
}

\footnotetext{
${ }^{1}$ Núcleo de Estudos de

Saúde Coletiva e

Laboratório de Economia

Política da Saúde,

Universidade Federal do

Rio deJaneiro. Av.

Brigadeiro Trompowisky

s/n, Praça da Prefeitura da

CidadeUniversitária,

21949-900, Rio de Janeiro

RJ. ligiabahia@terra.com.br

2 Núcleo de Estudos de

Saúde Coletiva da UFRJ.

${ }^{3}$ Instituto de Economia da

UFRJ eLaboratório de

Economia Política da Saúde

daUFRJ.
}

Abstract Several studies based on the 1998 PNAD Health Supplement data have shown significant differences in socioeconomic standards, access to and use of health services among population segments covered or not by health plans. Seeking to improve our knowledge on the structure and dynamics of the market of health plans and insurances in Brazil, we sought first to identify and then to highlight the different demands. To this effect, we conceived a typology of health plans on the basis of theinstitutional origin of the demands and on the relation between insurance and health plan holders and their dependents for three major categories: private employer plans, public employer plans and individual plans. Age, socioeconomic patterns and health conditions vary according to different health plan profiles, suggesting distinct patterns of demands, markedly between private employer and individual plans. Key words Private health plans, Insurance health plans, Demand segmentation, Health plan typology
Resumo A partir dos dados do Suplemento Saúdeda PNAD 1998, diversos estudos evidenciaram a presença de diferençassignificativasnos padrões socioeconômicos, acesso, utilização de serviços de saúdee con di ções de saúde entre os segmentos populacionais cobertos e não cobertos por planos de saúde. Buscando contribuir para aprimorar o acervo dos conhecimentos sobre a estrutura e a dinâmica do mercado de planos e seguros de saúde no Brasil, admite-see procura-se evidenciar a heterogeneidade dessas demandas. Para tanto, foi definida uma tipologia deplanos desaúde, fundamentada nas origens institucionais das demandas e, subseqüentemente, pelas relações de dependência com titulares de planos e seguros de saúde, integrada por três categorias princi pais: os planosempresariais privados, os planos empresariais públicos e os planos individuais. As características etárias, socioeconômicas ede condições de saúde variam segundo os diferentestipos de plano, sugerindo a presença de padrões distintos de demandas, especialmente entreos planos empresariaisprivados eos planos individuais.

Palavras-chave Planos privados de saúde, Segurosprivados de saúde, Segmentação da demanda, Tipologia de planos de saúde 


\section{Introdução}

Ao longo dos anos, o crescenteinteresse pelo tema das relações entre o público e o privado no sistema de saúde brasileiro contribuiu para ampliar o conhecimento sobre diversas dimensões das complexas articulações entre as instituições governamentais e as empresas privadas envolvidas com o financiamento, prestação de serviços e gestão de esquemas assistenciais para segmentos populacionais específicos. Uma das maiores lacunas para o exame das inter-relações entre a demanda e a oferta que conformam o denominado mercado de planos e seguros de saúde consistia na ausência de bases de dados populacionais sobre a cobertura de planos e seguros de saúde.

A inclusão de perguntas sobre coberturas de planos e seguros de saúde no Suplemento Saúde da PNAD 1998 estimulou a elaboração de análises acuradas sobre as tensões entre a universalização e a segmentação do sistema de saúde brasileiro1, 2, 3. Inspirou também a inserção de perguntas sobre as condições diferenciadas de cobertura e formas de pagamento de serviços em pesquisas de abrangência nacional, como a Pesquisa Mundial de Saúde no Brasil (2003) e o Inquérito Domiciliar sobre Comportamentos de Risco e Morbidade Referida de Doenças e Agravos não Transmissíveis (2002-2003). Informações detal hadas sobre as coberturas de planos de saúde, condições de saúde e utilização de serviços da população idosa também foram extraídas do estudo prospectivo iniciado a partir de 2001 na cidade de Bambuí, M inas Gerais, e no inquérito de saúde de jovens e idosos da Região M etropolitana de Belo Horizonte, realizado em 2003.

As análises dessas pesquisas sinalizam diferenças significativas sobre as condições socioe cononômicas, acesso e utilização de serviços para os segmentos cobertos e não cobertos por planos de saúde, inclusive em relação a ações e cuidados preventivos. Lima-Costa ${ }^{4}$ constatou que todos os serviços preventivos de saúdeforam significativamente mais freqüentes entre os filiados a planos e seguros de saúde, independentemente do sexo e da escolaridade. Viacava et al. ${ }^{5}$ demonstraram que mesmo quando ajustadas por idade, sexo, número de utensílios domésticos, escolaridade, porte do município de residência e autopercepção do estado de saúde, a probabilidade dos segmentos cobertos por planos e seguros de saúde de utilizar serviços de saúde é 34\% maior do que a dos não cobertos.

Entre os desdobramentos das investigações sobre a segmentação no sistema de saúde brasileiro, situam-se os estudos que apontam a pre- sença de uma estratificação interna ao mercado de planos e seguros. Teixeira ${ }^{6}$ enfatiza que os planos destinados a estratos de menor status socioocupacional, cujo valor é cerca de 20 vezes menor do que os acessíveis para os situados em cargos executivos, não podem ser considerados como produtos substitutos. Nesse sentido, o detal hamento das conexões entre as redes de empresas empregadoras, empresas de planos eseguros e prestadores de serviços de saúdeéfundamental para a compreen são dos circuitos diferenciados envolvidos com 0 atendimento de demandas diferenciadas, em termos de inserção socioocupacional, e não nas necessidades de saúde.

Buscando contribuir para o debate sobre a complexidade da segmentação das demandas e da oferta no sistema de saúde brasileiro, elaborou-se, a partir dos dados do Suplemento Saúde da PNAD 1998, uma tipologia baseada na origem institucional enas formas de financiamento dos planos de saúde. Com base nessa tipologia e em outros resultados da PNAD, questionou-se a noção da presença disseminada de fal has demercado na assistência suplementar à saúde. A magnitude do segmento de pessoas cobertas por planos originados de uma "estratificação passiva", isto é, decorrentes dos contratos estabel ecidos no mercado de trabal ho, quer no setor privado quer no público, supera a do integrado pelos clientes dos planos individuais. Dessas evidências emanaram recomendações sobre restrições ao uso indiscriminado dos conceitos de assimetria de informações, seleção adversa e risco moral para explicar a estrutura e a dinâmica do mercado de planos e seguros de saúde no Brasil e, em conseqüência, subsídios para o debate sobre a reorientação de determinados pressupostos das políticas públicas de regulação.

Dado o patamar mais elevado de conhecimentos sobre a importância da organização das demandas para o mercado de planos e seguros de saúde e a inclusão das perguntas sobre essas coberturas no Suplemento Saúde da PNAD 2003, o presente artigo examina, a partir da mesma tipologia, publicada por Bahia et al. ${ }^{7}$, a distribuição dos diferentes tipos de planos e o correspondente perfil das características demográficas, socioeconômicas e familiares, e das condições de saúde desses segmentos populacionais. A possibilidade da comparação de informações sobre as duas pesquisas faculta testar a consistência da tipologia elaborada anteriormente e ressaltar os padrões diferenciados das coberturas por planos e seguros de saúde. 
O Suplemento Saúde da PNAD e o acesso a informações sobre as demandas de planos eseguros de saúde

Os inéditos dados sobre cobertura dos planos e seguros de saúde registrados pela PNAD 1998 permitiram o exame do mercado de planos e seguros de saúde pelo ângulo da composição de suas demandas. Até então as informações sobrea organização de esquemas assistenciais adicionais aos disponíveis a toda à população, e preteritamente aos vinculados à Previdência Social, eram extraídam de fontes de empresas de planos e seguros de saúde, isto é, daqueles que os ofertam (comercializam).

0 exame da base de informações sobre a demanda de planos e seguros de saúde iluminou características do mercado de planos e seguros de saúde até então pouco exploradas, tal como: a existência de significativo contingente populacional coberto por planos de servidores civis estaduais e municipais e militares. A possibilidade de relacionar as coberturas dos planos com características do trabalho dos titulares permitiu a construção de uma tipologia de planos de saúde baseada na inserção socioocupacional. As informações individualizadas permitiram, pela primeira vez, estimar a composição do universo de brasileiros vinculados a planos e seguros de saúde, incluindo aqueles adscritos ao mercado de "planos particulares públicos".

A mobilização de abordagens baseadas nas características e estratégias de organização das demandas contribuiu para o avanço de duas ordens de hipóteses interligadas. 0 primeiro conjunto de hipóteses concerne à presença de elementos mais complexos do que o pertencimento ou não a determinadas faixas de renda. Ou seja, a noção muito difundida no Brasil de que todos os planos de saúde são, tal como qualquer objeto de consumo, "livremente adquiridos no mercado", é deslocada por acepções mais realistas amparadas na importância das estratégias corporativistas parajustificar parcela considerável das coberturas dos planos de saúde. Isso não significa desconhecer o poder explicativo isolado da variável renda em relação à cobertura ou não dos planos de saúde. Mas, sim, jogar luzes sobre a vigência de mecanismos associativos-institucionais interpostos nas estimativas de elasticidade da demanda por planos e seguros de saúde. A segunda ordem de considerações é um desdobramento do primeiro conjunto de hipóteses. Refere-seà perspectiva de balizar as relevantes diferenças entre os planos, tais como: distribuição etária, núme- ro de dependentes, status de saúde a partir de categorias analíticas da demanda, enão pelos limites e franquias dos contratos de planos e seguros. 0 que, por sua vez, reitera que 0 acionamento e a preservação de estratégias corporativistas fragmentadas e, portanto, diferenciadas quanto ao poder de obtenção de benefícios, estão associados não apenas ao fato de estar ou não coberto por planos e seguros de saúde, mas também à el egibilidade, abrangência e qualidade dessas coberturas.

\section{Procedimentos metodológicos}

A Pesquisa Nacional por Amostragem Domiciliar é planejada para ser um sistema de pesquisas por amostra probabilística de domicílios, de abrangência nacional, para atender a diversos propósitos relativos às áreas demográfica, de saúde, consumo alimentar e nutrição, condições de habitação e equipamentos domésticos, educação e cultura, trabalho e nível econômico do domicílio. Desde o início da PNAD, os temas eleitos para serem pesquisados, de forma permanente, através da Pesquisa Básica, foram habitação etrabalho, associados a al gumas características demográficas, educacionais e de rendimento. Eventualmente outros assuntos foram agregados à pesquisa básica, mas sem adquirir o caráter de permanência. Em 1998 e em 2003, foi aplicado um questionário suplementar, objetivando conhecer os padrões de consumo de serviços de saúde da população brasileira, à exceção da zona rural da região Norte.

A amostra da PNAD é selecionada em três estágios sucessivos. No primeiro estágio, são selecionados os municípios; no segundo, os setores; e, no último, as unidades domiciliares. Antes de se iniciar os estágios de sel eção da amostra, os municípios são separados em dois conjuntos. No primeiro são relacionados os municípios que, em decorrência do tamanho da sua população ou de alguma característica de importância, participam, necessariamente, da amostra. Estes foram classificados como auto-representativos. No segundo conjunto ficam os demais municípios, que passam por um processo de seleção, designados de "não auto-representativos".

O Suplemento Saúde possui dois subconjuntos de perguntas sobre as características dos planos desaúde. 0 primeiro éconformado por questões relativas ao financiamento dos planose, portanto, à natureza jurídico-institucional das instituições responsáveis pela contratação das cober- 
turas. 0 segundo está voltado para explorar as coberturas, considerando variações nos padrões de acesso mediado pelos planos ou seguros de saúde às redes de serviços. Em ambos os subconjuntos, muitas das perguntas foram dirigidas exclusivamente ao titular do plano.

As respostas referentes ao primeiro grupo de interrogações permitem, a partir da extensão de características dos planos dos titulares aos dependentes e da análise e agregação de variáveis originais da PNAD, cotejar uma "estratificação passiva" dos segmentos populacionais cobertos (planos empresariais) com uma possível estratificação dos riscos decorrente da demanda ativa por planos e seguros de saúde (planos individuais). Propiciam também uma estimativa mais realista da pouco visível cobertura por planos de saúde, administrados diretamente por instituições públicas, quenão estão sujeitos, por definição legal, ànormatização da Agência N acional deSaúdeSuplementar (ANS).

Uma vez que apenas os titulares responderam a determinadas perguntas sobre as características dos planos, procedeu-se à extensão da tipologia aos dependentes moradores do mesmo domicílio, através da vinculação do código do dependente ao de seu respectivo titular. Esse ajuste metodológico pressupõe que titulares e dependentes moradores dos mesmos domicílios de residência estejam cobertos pelos mesmos planos de saúde. A par da desconsideração da existência de variações entre o plano do titular e de seus dependentes, a impossibilidade de capturar as origens das demandas dos planos dos dependentes de titulares de outros domicílios remetequestionamentos à fidedignidade das estimativas sobre a distribuição das demandas por planos e seguros de saúde. Por outro lado, a configuração de um banco de dados so bre as características sócio-demográficas e de saúde do universo da população coberta por planos de saúde é essencial às análises de um mercado cujo número de dependentes é maior do que 0 de titulares ( 1,5 vez em 1998 e 1,25 em 2003).

Com base na vinculação de titulares e dependentes, as demandas por planos e seguros de saúde foram desagregadas segundo a origem institucional e o financiamento das coberturas em cinco categorias:

1) Sem plano de saúde.

2) Planos individuais, definidos pelas condições do pagamento do contrato de cobertura ser efetuado pelo titular, por outro morador do domicílio ou por pessoa não moradora do domicílio diretamente ao plano.
3) Planos empresariais públicos, classificados a partir da condição na ocupação ativa ou inativa do titular, que tenha declarado estar coberto por plano de instituição de funcionário público cujo vínculo ativo ou emprego anterior seja no setor público, nas esferas estadual ou municipal ou para os servidores militares nas mesmas condições.

4) Planos empresariais privados, determinados pela conjugação das condições do pagamento do contrato de cobertura ser efetuado pelo titular ou somente pelo empregador, através do emprego atual ou do anterior e da condição de atividade no setor público na esfera federal dos servidores civis e dos empregados no setor privado.

5) Planos de dependentes com titulares fora do domicílio, definidos pelas condições do pagamento do contrato ser efetuada por pessoa não moradora da unidade domiciliar.

Esta última categoria, para a qual as restrições de informações da PNAD sobre as características do plano são incontornáveis, integra a tipologia por representar um subgrupo populacional diferenciado do restante da população. $\operatorname{Re}-$ sumidamente, segundo os dados da PNAD 2003, os dependentes de titulares que moram em outros domicílios estão concentrados nas faixas etárias mais avançadas (20,3\% desses dependentes têm 65 anos ou mais, em contraste com apenas $6,6 \%$ de indivíduos nesta faixa etária na população total ), e apenas $55,6 \%$ se declaram sem morbidade relativamente aos $70,1 \%$ que se consideram hígidos na população em geral. À primeira vista, esse subgrupo apresenta características sugestivas da contratação individual de planos de saúde. Porém, é plausível supor a incidência de diferentes formas institucionais e de contratação em seu interior, pois $25,8 \%$ dos dependentes de titulares que moram em outros domicílios estão vinculados aos planos de instituições de servidores públicos.

Aproximadamente, um milhão e oitocentos mil pessoas cobertas por planos de saúde não foram categorizadas por essa tipologia. Essas perdas compreendem cerca de um milhão de pessoas, cobertas por planos financiados através de outras modalidades de pagamento, definidas pelo não enquadramento nos itens anteriores, como o financiamento compartilhado entre todos os moradores, entre moradores enão moradores do domicílio, somadas àquelas não classificadas devido a informações incompletas, erros e inconsistências relativas às variáveis definidoras das categorias de planos. 


\section{Resultados}

Os resultados iniciais, referidos exclusivamente ao plano único ou ao plano principal do titular, registram a cobertura através de planos de saúde, em 2003, para cerca de 43,2 milhões de pessoas ( $24,6 \%$ do total da população), sendo 34,2 milhões de brasileiros vinculados aos planos privados ( $79,0 \%$ do total de cobertos) e os restantes 9 milhões a planos de saúde de instituições de assistência aos servidores públicos (estaduais ou municipais) e militares. Comparativamente a 1998, as informações sobre essas características mantiveram-se quase inalteradas, uma vez queo aumento na proporção do contingente de clientes de planos privados de 75\% em 1998 para $79 \%$ em 2003 pode ser atribuído à mudança de classificação dos cobertos por planos de servidores civis de instituições federais. A alteração mais expressiva sobre as origens do financiamento do plano do titular concentra-sena distribuição das proporções entre os planos empresariais - financiados integral ou parcialmente pelo empregador e individuais - integralmente financiados pelas famílias. Em 1998, 60\% dos titulares estavam vinculados a planos empresariais, eem 2003 essa proporção foi de 50,1\% 8,9.

No que diz respeito às características das formas de organização do acesso às redes assistenciais dos planos e seguros, a análise revelou que $91,7 \%$ dos titulares de planos e seguros de saúde dispunham de coberturas através de listas de prestadores de serviços credenciados, enquanto apenas $31,7 \%$ estavam vinculados aos planos que facultam o reembolso. Esses resultados evidenciam não somente uma superposição do credenciamento com o reembolso para uma pequena parcela dos titulares cobertos, mas, sobretudo, a inequívoca predominância das formas gerenciadas da oferta e da demanda de serviços relativamente à livre escolha, típica dos seguros privados tradicionais. As coberturas dos planos, segundo a percepção dos entrevistados, não são restritas no que tange ao regime de atendimento e abrangência geográfica. 0 atendimento ambulatorial estáincluído em 98,5\% dos planos, os exames complementares em $96,6 \%$ e as internações hospitalares em $92,1 \%$. Por outro lado, apenas $7,5 \%$ abrangem 0 acesso aos medicamentos de uso ambulatorial.

Tais padrões mais gerais de coberturas dos planos são semelhantes aos de 1998, exceto em relação à elevação da proporção de $21,4 \%$ para $24,6 \%$ dos planos que incluem o co-pagamento e à diminuição das coberturas dos planos que incluem assistência odontológica, de 31,2 para $28,6 \%$. Conjugando-se esses resultados, depreende-se que não há uma grande variação do escopo das coberturas dos planos. Nota-se tão somente a presença de um contingente de pessoas vinculadas a planos queseriam mais pródigos em função da previsão do reembolso e as baixas coberturas via plano de saúde para assistência odontológica e medicamentos. A discreta elevação da proporção do co-pagamento nos planos de saúde pode ser atribuída à intensificação do uso de alternativas de repasse do aumento de custos assistenciais para os clientes, no contexto da regulação governamental do reajuste de preços.

No entanto, a estratificação dos padrões de coberturas pode ser apreendida pela análise do tipo de acomodação para internação, um dos fatores definidores do padrão do plano no Brasil, que foi incluída na PNAD 2003. Na percepção dos entrevistados, apenas 42,8 dos planos garante acesso a quarto privativo durante a vigência de internações hospitalares. Portanto, a maior parte dos planos estaria representada pelos de nominados "planos básicos".

Quando referidos ao conjunto da população, à distribuição dos planos baseada no status socioocupacional e na origem do financiamento, observa-se que entre os $24,6 \%$ de cobertos predominam os planosempresariais privados (10\%) e planos individuais (8,\%). Apenas 3,6\% dos entrevistados possuem plano tipo empresarial público e aproximadamente 3\% não tiveram a tipologia do plano desaúde classificada (Figura 1).

0 perfil de distribuição entre os planos cujas demandas estão diretamente relacionadas com a inserção do titulares no mercado de trabalho e com o pagamento direto do plano às empresas que os comercializam éanálogo ao de 1998. Destaca-se um pequeno incremento da proporção dos planos individuais, de 29,8\% em 1998 para $32,6 \%$ em 2003. Essa mudança decorreu principalmente da diminuição da proporção dos planos empresariais públicos (17\% em 1998 e 14,6\% em 2003), uma vez quea importância dos planos empresariais privados manteve se relativamente estável nas duas pesquisas: 41,6\% em 1998 e 40,6\% em 2003 (Tabela 1).

A distribuição por idade e sexo, segundo 0 tipo de plano, está representada por pirâmides na figura 2 e pelos indicadores na tabela 3 . A representação por pirâmides constitui uma importante estratégia gráfica, na medida em que permite a visual ização e a comparação da estrutura etária de diferentes grupos populacionais em certo momento ${ }^{10}$. 
As quatro pirâmides apresentam padrões distintos. 0 segmento populacional sem plano desaúde apresenta um padrão típico de população jovem em processo recente de envelhecimento, caracterizado pelo estreitamento da base e um relativo aumento do ápice da pirâmide. Este padrão se aproxima da população brasileira, tendo em vista que representa, aproximadamente, $75 \%$ dos entrevistados.

Os demais grupos, todos cobertos por planos de saúde, apresentam-semais "envel hecidos" quando comparados ao grupo sem plano de saúde, porém, com algumas variações. A pirâmide relativa ao grupo populacional coberto por planos de saúde do tipo empresarial privado mostra um predomínio das faixas etárias entre 25 e 49 anos, sempre mais expressivo na população feminina. D estaca-se ainda a participação relativa das faixas etárias jovens, em particular daquelas com idade inferior a 15 anos, embora com menos intensidade quando comparada ao grupo sem plano. Em contrapartida, a parcela de indivíduos com idades mais elevadas - igual ou superior a 55 anos - mostrou-se bastante reduzida. Trata-se, pois, de uma população envel hecida - quando comparada àquela sem plano -,

Figura 1

Tipos de planos de saúde classificados a partir da PNAD 2003 - Brasil.

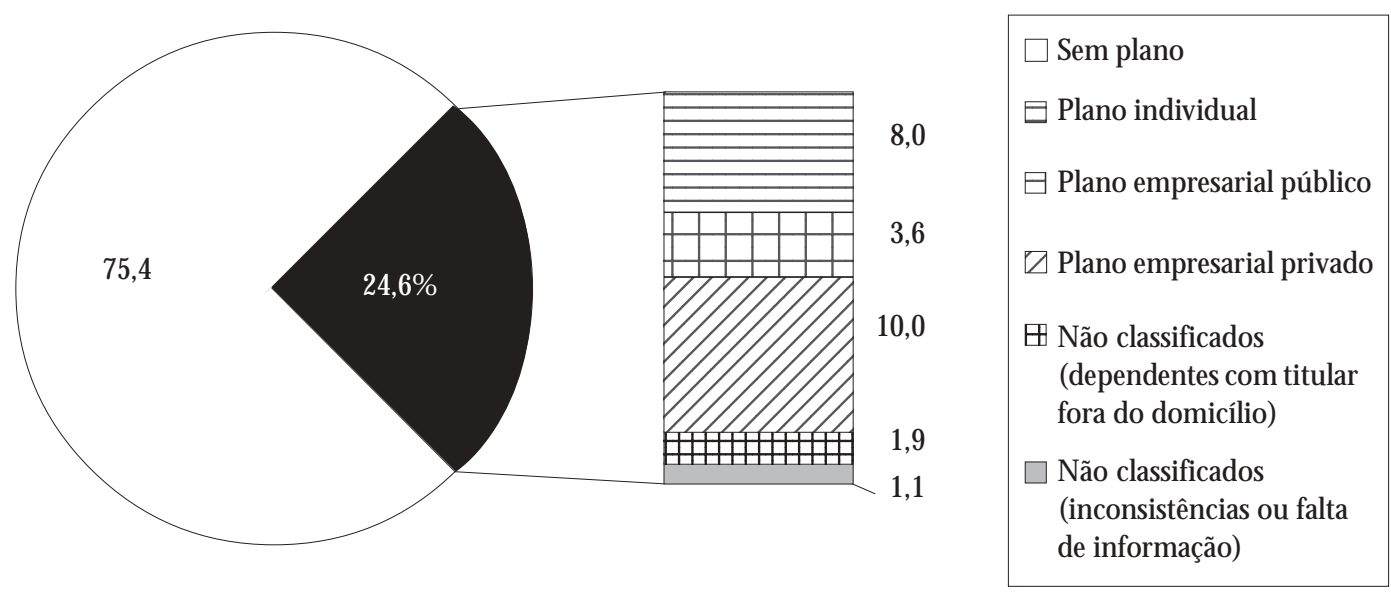

Tabela 1

Distribuição dos indivíduos segundo plano de saúde e tipo a partir das PNAD's de 1998 e 2003 - Brasil.

\begin{tabular}{|c|c|c|c|c|c|}
\hline \multirow{2}{*}{ Plano de saúde e tipo a parttir da PNAD } & \multicolumn{2}{|c|}{ PNAD 1998} & \multicolumn{2}{|c|}{ PNAD 2003} & \multirow{2}{*}{$\begin{array}{c}\text { Variação } \\
\text { 1998-2003 }\end{array}$} \\
\hline & $\mathrm{N}$ & $\%$ & $\mathrm{~N}$ & $\%$ & \\
\hline Indi víduos sem plano de saúde & 119.497 .410 & 75,5 & 132.771 .852 & 75,4 & $11,1 \%$ \\
\hline Indivíduos com plano de saúde & 38.680 .669 & 24,5 & 43.215 .760 & 24,6 & $11,7 \%$ \\
\hline Plano de saúde individual & 11.537 .790 & 29,8 & 14.076 .949 & 32,6 & $22,0 \%$ \\
\hline Plano empresarial público & 6.591 .518 & 17,0 & 6.316 .550 & 14,6 & $-4,2 \%$ \\
\hline Plano empresarial privado & 16.095 .546 & 41,6 & 17.519 .247 & 40,5 & $8,8 \%$ \\
\hline Dependentes com titular fora do domicílio & 2.491 .729 & 6,4 & 3.417 .423 & 7,9 & $37,2 \%$ \\
\hline Não classificados & 1.964 .086 & 5,1 & 1.885 .591 & 4,4 & $-4,0 \%$ \\
\hline População estimada segundo a PNAD & 158.178 .079 & 100,0 & 175.987 .612 & 100,0 & $11,3 \%$ \\
\hline
\end{tabular}

Nota: as percentagens dos tipos de planos de saúde se referem ao total de indivíduos com plano. 
porém, à custa, principalmente, das faixas etárias adultas em idade produtiva, ao longo das quais a cobertura por planos de saúde do tipo empresarial privado se faz presente.

A pirâmide relativa ao segmento coberto por planos empresariais públicos também mostraum predomínio das faixas etárias entre 25 e 49 anos, mas apenas entre a população feminina. Embora importante, a participação relativa das faixas etárias jovens mostra-se menos expressiva do que no segmento sem plano, particularmentea de crianças com idade inferior a 10 anos, em ambos os sexos. Já a parcela de indivíduos com idades acima de 54 anos, e mesmo aqueles com 65 ou mais anos, mostrou-se semel hante- por vezes atémaior - do que a observada entre aqueles sem cobertura por planos de saúde. Este padrão é indicativo de uma população mais envelhecida que aquela coberta por planos empresariais privados, porém com contingentes jovens ainda expressivos, e uma reduzi da participação relativa das faixas etárias produtivas - particularmente no sexo masculino - , aumentando assim a razão entre as faixas dependentes (jovens eidosos) eaquelas em idades produtivas. A elevada proporção de ido- sos permite supor que a cobertura por planos do tipo empresarial público estende-se além do período produtivo, diferentemente da modalidade empresarial privado.

O segmento coberto por plano de saúde individual é o mais envelhecido entre todos os grupos populacionais por ora analisados. A sua pirâmide mostra uma base estreita - evidenciando uma reduzida participação das faixas deidades jovens na composição da população - e um ápice mais largo, quando comparada à da população sem plano. Destaca-se também, a expressiva participação da população adulta. A partir dos vinte anos, a pirâmide mostra-se marcadamente assimétrica, indicando uma maior participação da população feminina à medida que a idade se eleva.

0 perfil da distribuição dos tipos de planos de saúde se repete segundo as áreas de residência, embora a cobertura nas áreas não auto-representativas seja bem inferior à encontrada nas regiões metropolitanas e áreas auto-representativas. A região Sudeste apresentou a maior cobertura de planos de saúde, 32,9\%, seguida das regiões Sul e Centro-O este, com 27,9\% e 24,7\%,

Figura 2

Pirâmides populacionais segundo tipos de planos de saúde a partir da PNAD 2003.

Sem plano de saúde

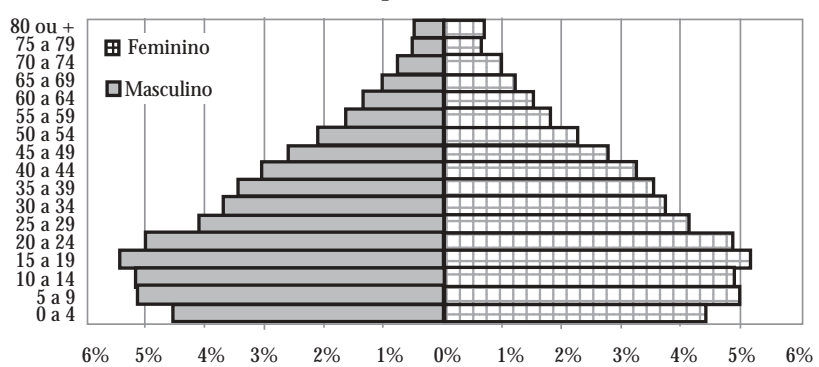

Plano de saúde empresarial público

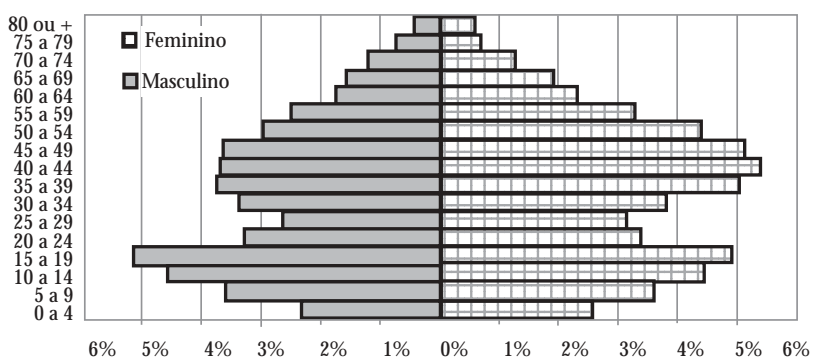

Plano de saúde individual

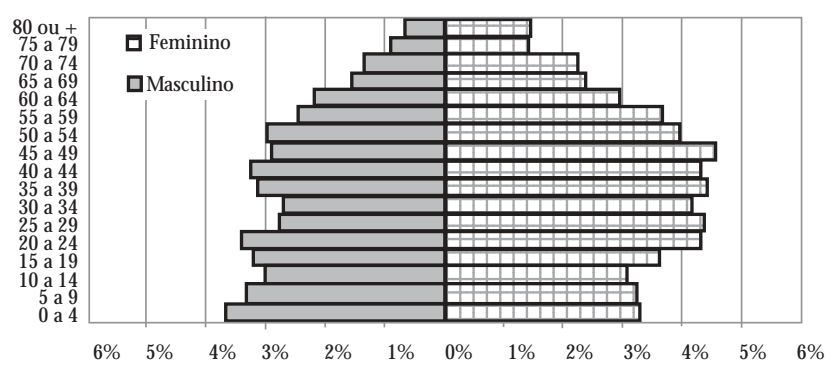

Plano de saúde empresarial privado

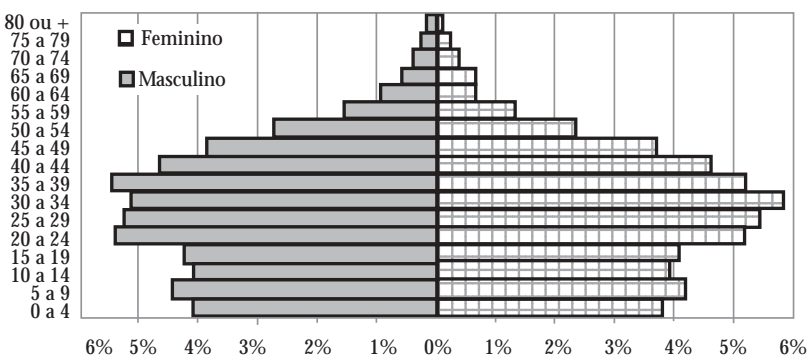


respectivamente. A distribuição dos entrevistados segundo o tipo de planos de saúde éheterogênea entre as regiões. Nas regiões Sudeste e Sul, economicamente mais favorecidas, predominam os planos empresariais privados e os planos individuais (respectivamente $14,8 \%$ e $11,1 \%$ na região Sudestee $10,6 \%$ e 8,9\% na região Sul). $\mathrm{Na}$ região Centro-Oeste, as coberturas dos planos empresariais privados $(7,9 \%)$, dos planos individuais $(6,6 \%)$ e dos planos empresariais públi$\cos (5,8 \%)$ mostraram-se próximas. Nas regiões Nordeste e N orte, as coberturas dos planos individuais (3,9\% e 4,5\%) foram semelhantes a dos empresariais privados (3,8\% e 4,7\%) (Tabela 2).

A tabela 3 sintetiza a distribuição de algumas variáveis socioeconômicas, segundo a tipologia de plano. Entre os entrevistados que declararam não ter plano, 27\% foram classificados como sem instrução (inclui as crianças); 46,8\% tinham de um a sete anos de estudo; e apenas 1,2\%, 15 anos ou mais. Entre aqueles que possuíam planos de saúde, por exemplo, do tipo individual, este valor foi de $16, \%$ (Figura 3). A média dos anos deestudo dos entrevistados quenão tinham planos de saúde foi 5,5, elevando-se para 9,1 entre os que possuíam plano do tipo empresarial privado, para 9,4 entre aqueles com plano empresarial público, e para 8,9 entre aqueles com plano individual.
A relação entre renda familiar per capita ecobertura de planos de saúde éainda mais evidente. Analisando as categorias extremas de renda, observa-seque $26,7 \%$ dos quenão possuíam plano de saúde concentram-se na categoria de renda abaixo de $R \$ 80,00$, ao passo que, aqueles que possuíam plano de saúde (classificados ou não), concentram-se $(71,1 \%)$ na categoria de renda de $\mathrm{R} \$ 300,00$ ou mais (Tabela 3 eFigura 4). O s valores medianos de renda familiar per capita segundo o tipo de plano foram de $R \$ 145,00$ para aque les sem plano; $\mathrm{R} \$ 595,00$ para plano individual; R\$ 466,60 para plano empresarial público; e $\mathrm{R} \$ 460,00$ para plano empresarial privado.

0 tipo de família predominante entre os entrevistados é aquele constituído pelo casal com filho(s) menor(es) de 14 anos (46,7\%). Esta característica se repete em todas as categorias de tipo de plano de saúde (Tabela 3).

As maiores coberturas de plano de saúde são de famílias formadas por casal com filhos maiores de 14 anos ou sem filhos. Os grupos pertencentes a famílias com mãe apenas apresentam menores coberturas do que aqueles com casal, em situações idênticas quanto à idade dos filhos. Independentemente de a família ser composta pelo casal ou apenas pela mãe, quando os filhos têm idades menores, iguais ou superiores a 14 anos, as coberturas são menores; quando os fi-

Tabela 2

Plano de saúde por tipologia segundo local de residência - Brasil, 2003.

\begin{tabular}{|c|c|c|c|c|c|c|c|c|}
\hline \multirow{3}{*}{ Local de residência } & \multicolumn{5}{|c|}{ Tipo de plano de saúde (\%) } & \multirow{3}{*}{$\begin{array}{c}\text { Sem plano } \\
\text { de saúde } \\
(\%)\end{array}$} & \multirow{2}{*}{\multicolumn{2}{|c|}{$\begin{array}{c}\text { Total de pessoas } \\
\text { (em milhares) }\end{array}$}} \\
\hline & \multirow{2}{*}{$\begin{array}{l}\text { Total c/ } \\
\text { plano }\end{array}$} & \multirow{2}{*}{$\begin{array}{l}\text { Indivi- } \\
\text { dual }\end{array}$} & \multirow{2}{*}{$\begin{array}{l}\text { Empres. } \\
\text { público }\end{array}$} & \multirow{2}{*}{$\begin{array}{l}\text { Empres. } \\
\text { privado }\end{array}$} & \multirow{2}{*}{ Outros* } & & & \\
\hline & & & & & & & $\mathrm{N}$ & $\%$ \\
\hline \multicolumn{9}{|l|}{ Área censitária** } \\
\hline Região metropolitana & 34,4 & 10,8 & 4,6 & 15,2 & 3,8 & 65,6 & 56.034 & 31,8 \\
\hline Auto-representativo & 31,5 & 10,9 & 4,2 & 12,4 & 4,0 & 68,5 & 37.896 & 21,5 \\
\hline N ão auto-representativo & 14,6 & 4,8 & 2,7 & 5,2 & 1,9 & 85,4 & 82.056 & 46,6 \\
\hline \multicolumn{9}{|l|}{ Região } \\
\hline Norte & 14,8 & 4,5 & 3,3 & 4,7 & 2,4 & 85,2 & 10.600 & 6,0 \\
\hline Nordeste & 12,1 & 3,9 & 2,4 & 3,8 & 2,0 & 87,9 & 49.950 & 28,4 \\
\hline Sudeste & 32,9 & 11,1 & 3,5 & 14,8 & 3,5 & 67,1 & 76.499 & 43,5 \\
\hline Sul & 27,9 & 8,9 & 5,0 & 10,6 & 4,4 & 72,1 & 26.366 & 15,0 \\
\hline Centro-Oeste & 24,7 & 6,6 & 5,8 & 7,9 & 4,4 & 75,3 & 12.570 & 7,1 \\
\hline
\end{tabular}

* Incluídos os dependentes com titular fora e aqueles com plano não classificados por falta de informação.

**Relacionam-se aos municípios que participaram do processo de seleção da amostra, podendo refletir a magnitude deles.

Os auto-representativos têm probabilidade 1 de pertencer à amostra e os não auto-representativos passam por um processo de estratificação e, em cada estrato, são selecionados com probabilidade proporcional à população. 
Ihos têm, todos, idades inferiores a 14 anos, as coberturas aumentam, e alcançam os maiores valores quando todos têm, no mínimo, 14 anos.

A fim de se simplificar e facilitar a interpretação dos resultados, as questões de morbidade da PNAD 2003 foram ligeiramente modificadas. A pergunta sobre auto-avaliação de saúde, originalmente em cinco níveis ( muito boa, boa, regular, ruim e péssima), foi dicotomizada em "boa" e"não-boa", por seconsiderar queuma auto-avaliação de saúde até regular deve traduzir uma auto-avaliação não satisfatória. As 11 doenças levantadas pelo questionário foram classificadas em três grupos, tentando-se retratar um certo grau de gravidade da situação de saúde referida pelo entrevistado. No primeiro grupo, ficaram dor nas costas etendinite; no segundo grupo, artrite, diabetes, bronquite, depressão e tuberculose; e, no terceiro grupo, câncer, doença no coração, doença renal e cirrose. 0 quadro 1 ilustra como foi definida a escala de mobilidade física para indivíduos com 14 anos ou mais, a partir das condições que foram objeto da pesquisa.

A tabela 4 mostra que a proporção de indivíduos sem morbidade referida émaior entreaqueles sem plano de saúde do que entre aqueles com plano empresarial privado - $72 \%$ e $70,6 \%$, respectivamente. A morbidade referida para tipo I e tipo II é maior nos clientes de planos empresariais públicos e individuais ( $37 \%$ e $35 \%$ ). M as não se pode afirmar uma associação causal entre 0 tipo de plano e o status de saúde, uma vez que as características individuais e familiares poderiam explicar - pelo menos parcialmente - não só a sua existência, como também a sua magnitude.

0 tipo de plano empresarial privado também apresenta a menor proporção de indivíduos que referiram doenças mais graves (4 \%) (Figura 5). Q uanto à auto-avaliação de saúde, há um predomínio da condição "boa”, tanto para aqueles com plano quanto para aqueles sem plano de saúde, respectivamente de $84 \%$ e $77 \%$. A condição de mobilidade "sem limitação" é mais freqüente no tipo de plano "empresarial privado". Os indivíduos sem plano de saúde e aqueles com plano "individual" e "empresarial público" apresentam uma distribui ção semel hante na escala de mobilidade utilizada (Figura 6).

Como seria esperado, há uma clara tendência de diminuição da autopercepção sobreas condições consideradas boas na faixa etária acima de 65 anos, independentemente da cobertura de planos e seguros de saúde. Mas, observa-se também que aqueles cobertos pel os planos e seguros de saúde relataram uma melhor autopercepção de seu estado de saúde, especialmente os que tinham doenças diagnosticadas. Para os que se declararam saudáveis, na faixa etária de 14 a 64 anos, não se observam diferenças relevantes sobrea autopercepção do estado de saúde para os cobertos ou não cobertos por planos e seguros de saúde. M as a boa percepção sobre o estado de saúde dos declarados hígidos vinculados a planos de saúde na faixa etária dos maiores de 65 anos é significativamente superior (cerca de $80 \%$ ) a dos sem planos (aproximadamente 60\%). Quando con-

Figura 3

Anos de estudo segundo tipologia de plano de saúde a partir da PNAD 2003 - Brasil.

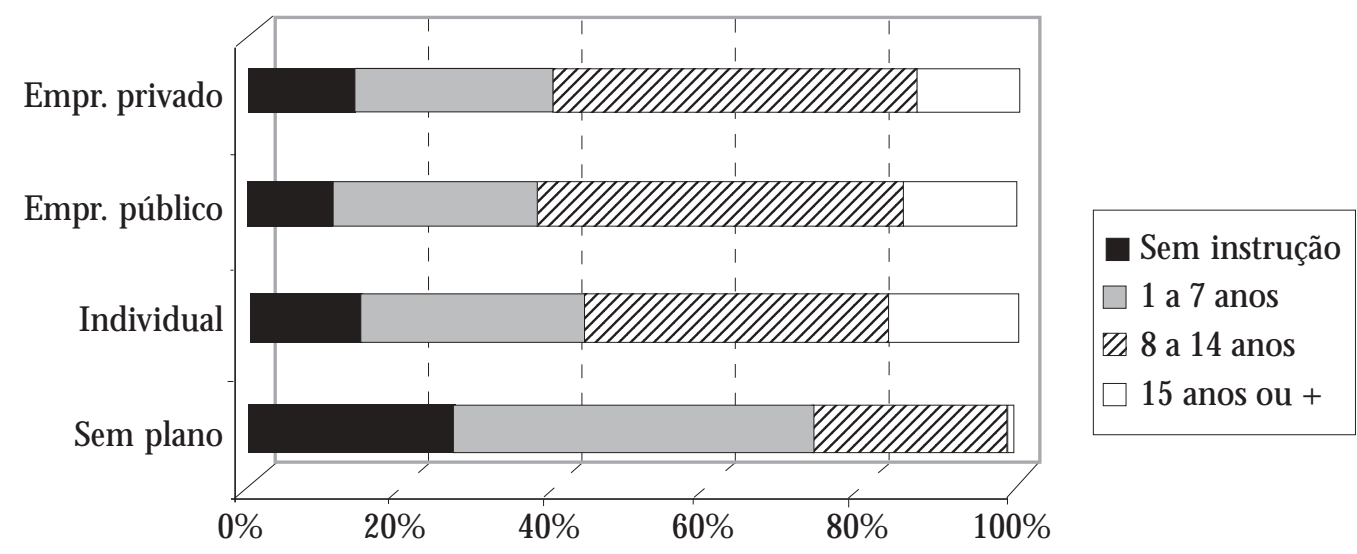


Figura 4

Renda familiar per capita segundo tipos de plano de saúde a partir da PNAD 2003 - Brasil.

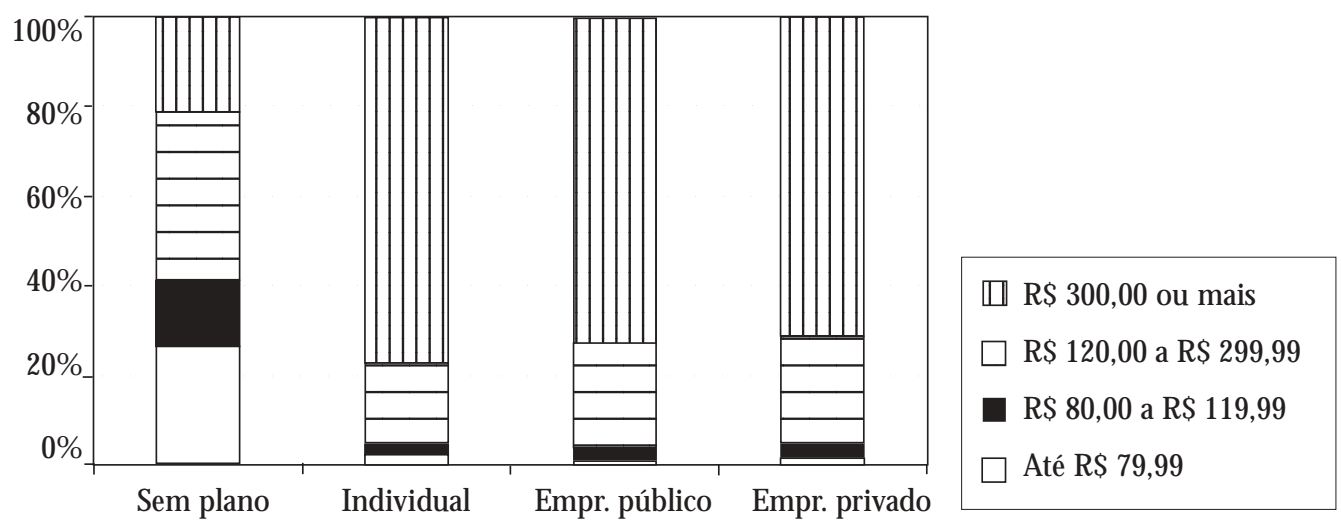

Tabela 3

Distribuição das variáveis socioeconômicas segundo tipos de planos de saúde a partir da PNAD 2003 - Brasil.

\begin{tabular}{|c|c|c|c|c|c|c|c|}
\hline \multirow{3}{*}{$\begin{array}{c}\text { Variáveis } \\
\text { socioeconômicas }\end{array}$} & \multirow{3}{*}{$\begin{array}{c}\text { Sem } \\
\text { plano de } \\
\text { saúde (\%) }\end{array}$} & \multicolumn{4}{|c|}{ Tipo de plano de saúde (\%) } & \multirow{2}{*}{\multicolumn{2}{|c|}{$\begin{array}{r}\text { Total de pessoas } \\
\text { (em milhares) }\end{array}$}} \\
\hline & & \multirow{2}{*}{$\begin{array}{l}\text { Total cl } \\
\text { plano* }\end{array}$} & \multirow{2}{*}{$\begin{array}{l}\text { Indivi- } \\
\text { dual }\end{array}$} & \multirow{2}{*}{$\begin{array}{l}\text { Empres. } \\
\text { público }\end{array}$} & \multirow{2}{*}{$\begin{array}{l}\text { Empres. } \\
\text { privado }\end{array}$} & & \\
\hline & & & & & & $\mathrm{N}$ & $\%$ \\
\hline \multicolumn{8}{|l|}{ Anos de estudo } \\
\hline Sem instrução & 27,0 & 14,3 & 14,4 & 11,2 & 13,9 & 41.927 & 23.8 \\
\hline 1 a 7 anos & 46,8 & 27,9 & 29,2 & 26,2 & 25,6 & 73.972 & 42,0 \\
\hline 8 a 14 anos & 25,0 & 43,8 & 39,7 & 47,6 & 47,4 & 52.049 & 29,6 \\
\hline 15 anos ou mais & 1,2 & 13,9 & 16,6 & 14,9 & 13,1 & 7.605 & 4,3 \\
\hline \multicolumn{8}{|l|}{ Renda familiar per capita } \\
\hline Até R\$79,99 & 26,7 & 2,4 & 2,2 & 1,0 & 1,5 & 35.755 & 20,8 \\
\hline 80,00 a 119,99 & 14,1 & 2,8 & 2,2 & 2,6 & 3,0 & 19.574 & 11,4 \\
\hline 120,00 a 299,99 & 38,4 & 23,6 & 18,5 & 24,0 & 25,7 & 59.865 & 34,8 \\
\hline 300,00 ou mais & 20,8 & 71,1 & 77,1 & 72,4 & 69,8 & 56.724 & 33,0 \\
\hline \multicolumn{8}{|l|}{ Tipo de família } \\
\hline Casal sem filhos & 8,8 & 11,6 & 13,7 & 10,8 & 9,3 & 16.760 & 9,5 \\
\hline Casal com pelo menos 1 filho $<14$ anos & 48,3 & 42,0 & 35,6 & 41,7 & 52,8 & 82.233 & 46,7 \\
\hline Casal com filhos $>14$ anos & 17,5 & 24,9 & 27,8 & 29,0 & 24,1 & 33.961 & 19,3 \\
\hline M ãe com pelo menos 1 filho $<14$ anos & 9,3 & 5,3 & 4,2 & 4,0 & 3,0 & 14.585 & 8,3 \\
\hline M ãe com filhos > 14 anos & 8,1 & 8,0 & 9,1 & 7,5 & 5,5 & 14.230 & 8,1 \\
\hline Outrostipos & 8,1 & 8,1 & 9,5 & 7,0 & 5,3 & 14.217 & 8,1 \\
\hline
\end{tabular}

Nota: as percentagens referem-se ao total de cada tipo de plano.

* Incluídos os dependentes com titular fora e aqueles com plano não classificados por falta de informação. 
Quadro 1

Definição da escala de mobilidade física tendo como critério as características de mobilidade física da PNAD 2003 para indivíduos com 14 anos ou mais.

\begin{tabular}{|c|l|}
\hline $\begin{array}{c}\text { Escala de } \\
\text { mobilidade }\end{array}$ & \multicolumn{1}{c|}{ Critério utilizado a partir das características de mobilidade da PNAD 98 } \\
\hline Muito limitada & $\begin{array}{l}\text { Não consegue ou tem grande dificuldade dealimentar-se, tomar banho } \\
\text { ou ir ao banheiro; ou não consegue realizar pelo menos uma das } 6 \text { outras } \\
\text { características de mobilidade física. }\end{array}$ \\
\hline Limitada & $\begin{array}{l}\text { Consegue fazer tudo, mas tem grande dificuldade em pelo menos uma } \\
\text { das características de mobilidade (excluída a que se refereà alimentação, } \\
\text { tomar banho ou ir ao banheiro). }\end{array}$ \\
\hline Pouco limitada & $\begin{array}{l}\text { Não tem nenhuma grande dificuldade, mas tem pequena em pelo menos } \\
\text { uma das características de mobilidade. }\end{array}$ \\
\hline Sem limitação & Não tem nenhum grau de dificuldadenas características de mobilidade. \\
\hline
\end{tabular}

Nota: características de mobilidade da PNAD 2003:

1) Alimentar-se, tomar banho ou ir ao banheiro; 2) andar cerca de 100 metros; 3) andar mais do que $1 \mathrm{~km} ; 4$ ) abaixar-se, ajoelhar-se ou curvar-se; 5) subir ladeira ou escada; 6) empurrar mesa ou realizar consertos domésticos; 7) correr, levantar objetos pesados, praticar esporte ou realizar trabal hos pesados.

\section{Tabela 4}

Distribuição das variáveis de morbidade referida, auto-avaliação de saúde e mobilidade física segundo tipos de planos de saúde a partir da PNAD 2003 - Brasil.

\begin{tabular}{|c|c|c|c|c|c|c|c|}
\hline \multirow{2}{*}{ Variáveis } & \multirow{2}{*}{$\begin{array}{l}\text { Sem plano } \\
\text { de saúde } \\
\text { (milhares) }\end{array}$} & \multicolumn{4}{|c|}{ Tipo de plano de saúde (em milhares) } & \multicolumn{2}{|c|}{$\begin{array}{c}\text { Total de pessoas } \\
\text { (em milhares) }\end{array}$} \\
\hline & & $\begin{array}{l}\text { Total c/ } \\
\text { plano }\end{array}$ & $\begin{array}{l}\text { Indivi- } \\
\text { dual }\end{array}$ & $\begin{array}{l}\text { Empres. } \\
\text { público }\end{array}$ & $\begin{array}{l}\text { Empres. } \\
\text { privado }\end{array}$ & $\mathrm{N}$ & $\%$ \\
\hline \multicolumn{8}{|l|}{ M orbidade referida } \\
\hline Sem morbidade & 95.453 & 27.877 & 8.427 & 3.966 & 12.379 & 123.331 & 70,1 \\
\hline . Doenças I (mais "crônicas”)** & 30.373 & 12.626 & 4.544 & 1.927 & 4.435 & 43.000 & 24,2 \\
\hline Doenças II (mais“graves")*** & 6.939 & 2.697 & 1.102 & 417 & 704 & 9.637 & 5,5 \\
\hline Auto-avaliação de saúde & 132.765 & 43.200 & 14.073 & 6.310 & 17.518 & 175.968 & \\
\hline Boa ou muito boa & 102.069 & 36.258 & 11.452 & 5.212 & 15.507 & 138.327 & 78,6 \\
\hline Regular, ruim ou muito ruim & 30.688 & 6.942 & 2.619 & 1.099 & 2.011 & 37.631 & 21,4 \\
\hline \multicolumn{8}{|l|}{ Escala de mobilidade física**** } \\
\hline . Muito limitada & 6.087 & 1.733 & 743 & 240 & 340 & 7.821 & 5,9 \\
\hline Limitada & 8.935 & 2.559 & 1.036 & 409 & 639 & 11.494 & 8,7 \\
\hline Pouco limitada & 11.203 & 4.076 & 1.522 & 681 & 1.299 & 15.280 & 11,6 \\
\hline Sem limitação & 70.855 & 26.051 & 8.222 & 3.819 & 11.399 & 96.908 & 73,7 \\
\hline
\end{tabular}

* Incluídos os dependentes com titular fora e aqueles com plano não classificados por falta de informação.

** Problemas na coluna, tendinite, artrite, diabetes, bronquite, depressão e tuberculose.

*** Câncer, doença do coração, doença renal e cirrose.

**** A penas para indivíduos com 14 anos ou mais. 
trolado pela morbidade percebida, os planos empresarial público eindividual apresentam padrões semelhantes (Figura 7).

\section{Discussão}

A principal diferença dos resultados dos dados sobre coberturas de planos de saúde baseados na PNAD e dos coletados e divulgados pela AN Séa relativa à magnitude dos segmentos vinculados a planos individuais e coletivos. Segundo a PNAD 2003, quando considerados dependentes e titulares, a proporção de planos empresariais de pelo menos 55\% (soma dos planos empresarial privado e planos empresarial público) é maior do que a aferida pela freqüência das respostas dos titulares sobrea origem do financiamento do plano, e mais próxima das informações divulgadas pelas próprias empresas de planos e seguros de saúde. Se tomarmos apenas o universo de planos regulados pela ANS, isto é, os empresariais pri-

Figura 5

M orbidade referida segundo tipos de plano de saúde a partir da PNAD 2003 - Brasil.

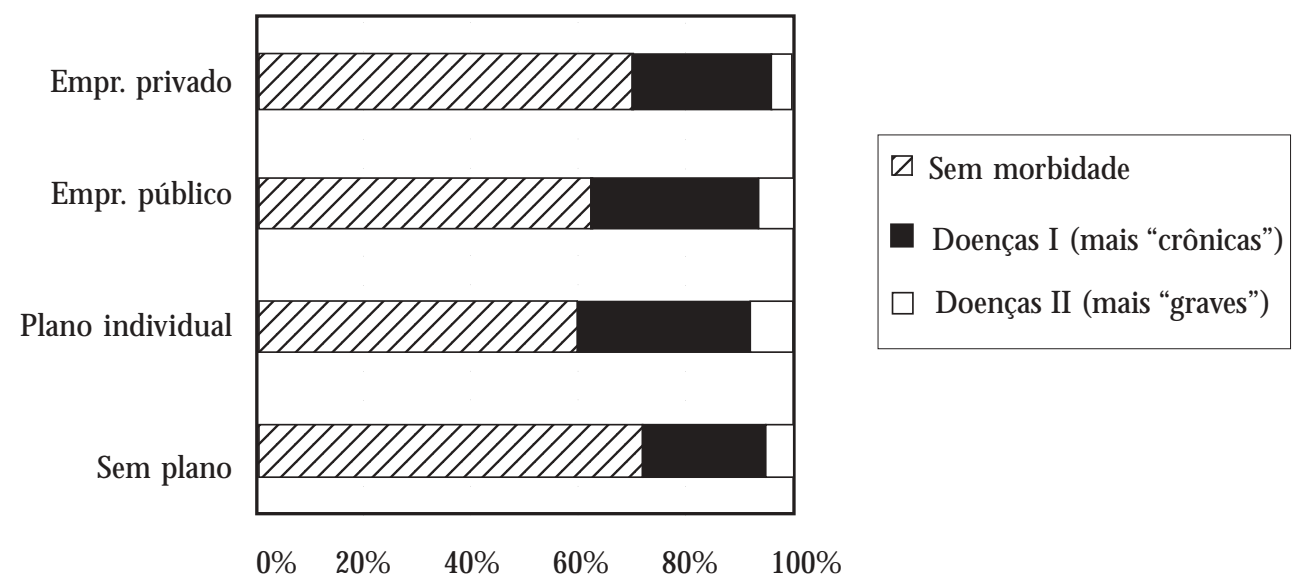

Figura 6

Escala de mobilidade física segundo tipos de plano de saúde a partir da PNAD 2003 - Brasil.

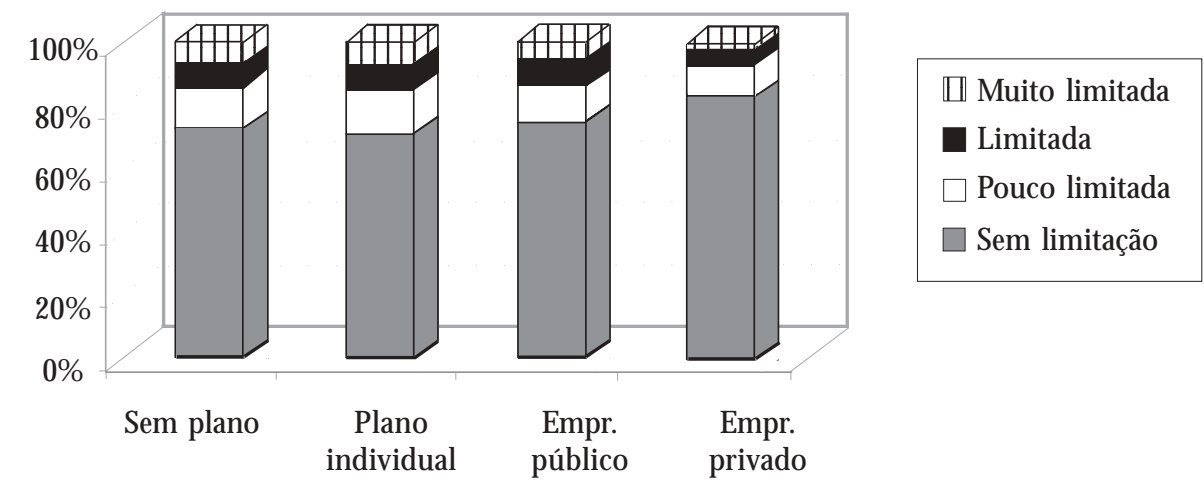


vados e os individuais, admitindo-se ainda quea totalidade dos planos de dependentes com titular fora do domicílio relaciona-se com empresas especializadas em planos e seguros de saúde privados ou empresas empregadoras de servidores civis pertencentes à esfera pública federal, a proporção de planos vinculados ao emprego atual ou anterior do titular seria de $60 \%$. M as ainda assim essa proporção não se coaduna totalmente com as informações divulgadas pela ANS, que contabiliza para os planos novos $75 \%$ deplanos coletivos (inclui planos que são financiados integralmente pelo empregado) e apenas $25 \%$ de individuais ${ }^{11}$.

Certamente, o fato de parte dos planos serem financiados pelo empregado e pelo empregador pode influenciar as respostas deinformantesnão titulares, emesmo de titulares que tendam a considerar muito elevados os descontos no salário como pagamento direto à empresa de plano de saúde. Por outro lado, as estimativas das proporções das coberturas dos planos "básicos" (que só garantem internação em acomodações coletivas) relativamente aos "superiores" são muito próximas daquelas reportadas, informalmente, por administradores de empresas de planos e seguros de saúde.

A participação relativa dos diferentes tipos de planos nos dois períodos, 1998 e 2003, categorizada por um duplo movimento - aumento das

Figura 7

Proporção de pessoas com boa ou muito boa auto-percepção de saúde, segundo morbidade referida e tipo de plano de saúde em indivíduos entre 14 e 64 anos e 64 anos ou mais, PNAD 2003.

Proporção de pessoas com boa ou muito boa auto-percepção de saúde, segundo morbidade referida e tipo de plano de saúde em indivíduos entre 14 e 64 anos, PNAD 2003 - Brasil.

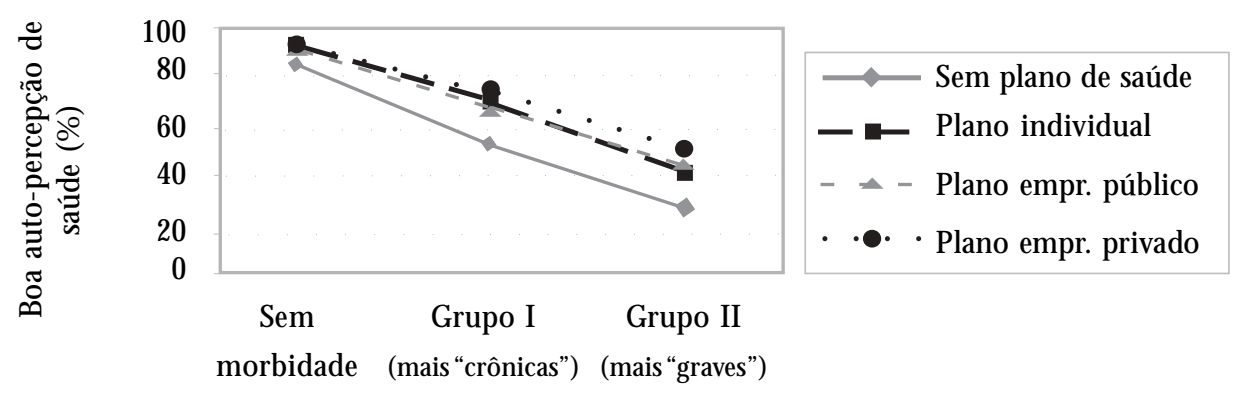

Morbidade referida

Proporção de pessoas com boa ou muito boa auto-percepção de saúde, segundo morbidade referida e tipo de plano de saúde em indivíduos com 64 anos ou mais, PNAD 2003 - Brasil.

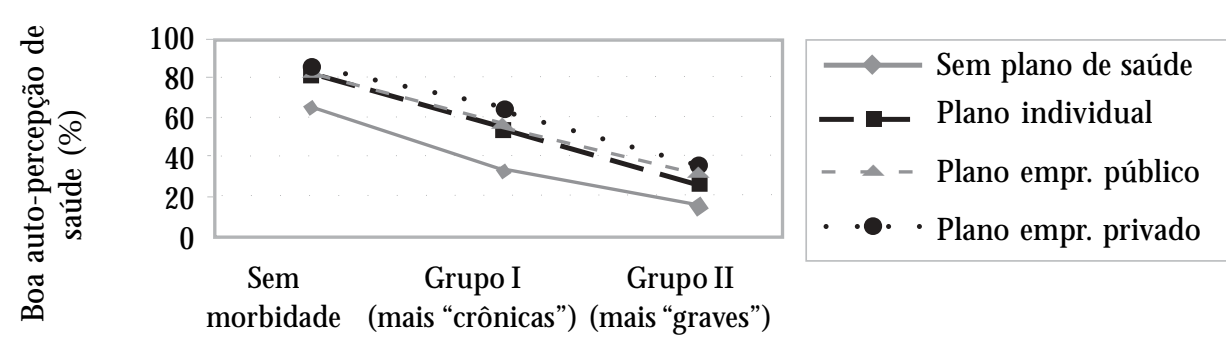

Morbidade referida 
coberturas por meio de uma discreta expansão dos planos individuais e ligeira retração dos empresariais públicos - não corresponde às tendências registradas pela ANS, que apontam no âmbito dos contratos novos, entre dezembro de 2000 e dezembro de 2003, uma estabilidade da proporção de planos individuais (27\% em dezembro de 2000 e $26 \%$ em dezembro de 2003) ${ }^{11}$.

Tampouco a diminuição relativa das coberturas dos planos empresariais públicos parece condizente com a dinâmica mais recente de expansão das demandas por planos e seguros de saúde. Na realidade, no período posterior a 2003, parece se delinear um movimento com sentido contrário, exemplificado pela criação de planos de saúde para os servidores do Rio de Janeiro e deBrasília, consubstanciados respectivamentena Lei Complementar 67, de 2003, e no Projeto de Lei $n=2.277$, de 2005.

\section{Considerações finais}

Procurando sintetizar o perfil dos indivíduos que tinham planos de saúde em 2003, isoladamente por cada atributo, pode-se dizer que são predominantemente mulheres, com idade mais avançada, mai or renda familiar per capita, maior escolaridade, pertencentes a famílias constituídas por casal sem filhos ou com filhos com pelo me nos 14 anos de idade, residentes nas regiões Sul ou Sudeste e em áreas metropolitanas. Este perfil foi mais típico para aqueles com plano individual em relação aos com plano empresarial privado, à exceção dos atributos relacionados ao local de residência.

De forma geral, o plano empresarial público apresentou um perfil intermediário entre os planos individual e empresarial privado, com relação a sexo, idadeetipo de família. Com relação à escolaridade, são mais próximosaos do plano empresarial privado. São bastantediferentes com relação à renda e ao local de residência: apresentam menor renda e uma distribuição menos heterogênea em relação à região de residência, me- nos concentrados na região Sudeste e nas áreas metropolitanas.

Por outro lado, foram levantadas al gumas características sociais e demográficas e pôde-se verificar que a aquisição de plano de saúde individual pode depender delas. Além da associação com a aquisição de plano de saúde, tais características também se relacionam às condições de saúde das pessoas. Nesse sentido, a complexidade que envolve a detecção e a quantificação das variáveis envolvidas com as demandas pelos contratos dos planos eseguros deveser analisada, necessariamente, de forma multivariada. Bahia et al. ${ }^{7}$ evidenciaram que renda e escolaridade são os principais fatores para aquisição de plano individual, sendo a morbidade referida um fator de menor relevância.

No entanto, isso não significa afirmar que a preservação das tendências de expansão dos planos individuais, evidenciadas pelas informações das PNAD 2003 por referência à de 1998, adicionadas ao possível crescimento dos planos empresariais de funcionários públicos estaduais e municipais, não desafiem a lógica de equilíbrio econômico-financeiro que presidiu a estruturação do mercado de planos eseguros de saúde no Brasil. A recente expansão das demandas individualizadas, especialmente após 2003, seja sob a forma de novas titularidades, quer como agregação a planos empresariais, mesmo que contraposta à retração da comercialização de planosindividuais por parte de al gumas seguradoras, parece acentuar as singularidades do mercado de planos e seguros de saúde no Brasil relativamente ao dos EUA, onde a proporção de planos individuais em 2004 foi de $9,3 \%{ }^{12}$. 0 elevado contingente de pessoas vinculadas a planos individuais e a reedição de redes de proteção patronais-familiares, moduladas pela retração relativa do mercado formal de trabalho, contrastam com as elevadas taxas de crescimento econômico que contextualizaram a emergência das empresas de planos privados de saúde, no Brasil da década de 1960. 


\section{Colaboradores}

L Bahia e RR Luiz participaram da conceituação, revisão bibliográfica e elaboração do artigo. C Salm tomou partena discussão dos marcos teóricos e análise dos resultados. AJ L Costa foi responsável pelo delineamento da metodologia e a estruturação do banco de dados. PL Kale colaborou com a seleção e a interpretação das informações. LT Caval canti contribuiu para a análise dosdados.

\section{Referências}

1. Farias LO, M elamed C. Segmentação de mercados da assistência à saúde no Brasil. Rev C S Col 2003; 8(2):585-98.

2. Santos MA, Gerschman S. As segmentações da oferta de serviços de saúde no Brasil - arranjos institucionais, credores, pagadores e provedores. Rev C S Col 2004; 9(3):795-806.

3. Andrade M V. Financiamento do setor de saúde suplementar no Brasil: uma investigação empírica a partir dos dados da Pnad/98. In: Werneck AJ, M ontone J, organizadores. Regulação e saúde: documentos técnicos de apoio ao Fórum de Saúde Suplementar de 2003. 1a ed. Rio de Janeiro: Ministério da Saúde/Agência Nacional de Saúde Suplementar. v. 3. p. 249-332.

4. Lima-Costa M F. Estilos de vida e uso de serviços de saúde entre adultos filiados ou não a plano privado de saúde (inquérito de saúde de Belo H orizonte). Rev C S Col 2004; 9(4):857-64.

5. Viacava F, Souza-Junior PRB, Szwarcwald CL. Cobertura da população brasileira com 18 anos ou mais por plano de saúde privado: uma análise dos dados da Pesquisa Mundial de Saúde. Cad Saúde Pública 2005; 21(Supl 1). p.119-28.

6. Teixeira A. M ercado eimperfeições do mercado: 0 caso da assistência suplementar. Texto para discussão; 2002. [M imeo].
7. Bahia L, Costa AJL, Luiz RR, Cavalcanti M LT. Segmentação e demanda dos planos e seguros privados de saúde: uma análise das informações da PNAD/98. Rev C S Col 2002; 7(4):671-86.

8. Brasil. Ministério do Orçamento e Gestão. Instituto Brasileiro de Geografia eEstatística. Pesquisa Nacional por Amostra de D omicílios: acesso e utilização de serviços de saúde 1998. Rio de Janeiro: IBGE; 2001.

9. Brasil. Ministério do Orçamento e Gestão. Instituto Brasileiro de Geografia eEstatística. Pesquisa Nacional por Amostra de Domicílios: acesso eutilização de serviços de saúde 2003. Rio de Janeiro: IBGE; 2005.

10. Laurenti R, Jorge MHPM, Lebrão ML, Gotlieb SLD Estatísticas de saúde. 2ª ed. São Paulo: E.P.U.; 1987.

11. Agência Nacional de Saúde Suplementar. Caderno de informações sobre beneficiários, operadoras e planos: edição novembro/2005 [acessado 2006 abr 12]. Disponível em: ttp://www.ans.gov.br/portal/site/consultas/ consultas.asp

12. U.S. Census Bureau. Current Population Survey. Annual Social and Economic Supplement. U.S. Census Bureau; 2005 [acessado 2006 mar 7]. Disponível em: http://www.census.gov/hhes/www/hlthins/historic/ index.html

Artigo apresentado em 29/03/2006

Aprovado em 18/04/2006

Versão final apresentada em 15/05/2006 